\title{
A single and novel natriuretic peptide is expressed in the heart and brain of the most primitive vertebrate, the hagfish (Eptatretus burgeri)
}

\author{
A Kawakoshi, S Hyodo, A Yasuda ${ }^{1}$ and Y Takei \\ Ocean Research Institute, University of Tokyo, Tokyo 164-8639, Japan \\ ${ }^{1}$ Suntory Institute for Bioorganic Research, Osaka 618-8503, Japan \\ (Requests for offprints should be addressed to A Kawakoshi; Email: kawakosi@ori.u-tokyo.ac.jp)
}

\begin{abstract}
In teleost fish and tetrapods, the natriuretic peptide (NP) family consists of ANP (atrial natriuretic peptide), BNP (brain natriuretic peptide) and VNP (ventricular natriuretic peptide) that are secreted from the heart, and C-type natriuretic peptide (CNP) that is found in the brain. However, CNP is the only NP identified in the heart and brain of elasmobranchs, suggesting that it is the ancestral type of the NP family and that ANP, BNP and VNP appeared later in the vertebrate phylogeny. To delineate more clearly the molecular evolution of this hormone family, we determined the sequence of NP molecule(s) in evolutionarily the oldest vertebrate group, the cyclostomes. We have cloned a novel NP cDNA from the heart and brain of hagfish, Eptatretus burgeri, using the RACE method and degenerate primers that amplify all known types of NP cDNAs. The novel NP, named EbuNP after the scientific name of this hagfish, appears to be the only NP in the heart and brain, as no other NP cDNAs were amplified even after specific removal of the cloned EbuNP mRNA from the mRNA pool, except for a minor alternatively spliced EbuNP cDNA with a truncated 3'-untranslated sequence. The EbuNP was equally similar to known NPs but was not considered to be a CNP because of the presence of a C-terminal tail sequence. The EbuNP gene was abundantly expressed in the cardiac atrium, ventricle, portal heart and brain but scarcely in the intestine; no expression was observed in the gill and kidney. Mass spectrometry of affinity-purified EbuNP in plasma, heart and brain revealed a 68 amino acid peptide circulating in the blood and stored in the heart, which is cleaved at the typical cleavage signal of a processing enzyme, furin, as observed in mammalian BNP. The C-terminal Gly residue was used for amidation as is the case in eel ANP. The immunoreactive EbuNP was not detected in the brain, suggesting the presence of a different processing form in the brain. These results show that the molecular evolution of the NP family in vertebrates is more complex than previously thought.
\end{abstract}

Journal of Molecular Endocrinology (2003) 31, 209-220

\section{Introduction}

Natriuretic peptides (NPs) are a hormone family that plays a pivotal role in cardiovascular and body fluid homeostasis in vertebrates (Brenner et al. 1990, Evans 1990, Farrell \& Olson 2000, Loretz \& Pollina 2000, Takei 2000). While the NP family consists of atrial natriuretic peptide (ANP), brain natriuretic peptide (BNP) and C-type natriuretic peptide (CNP) in mammals and amphibians, ANP, VNP (ventricular natriuretic peptide) and CNP occur in teleost fish. ANP, BNP and VNP, which have a C-terminal 'tail' sequence of variable length that extends from the intramolecular ring structure formed by a disulfide bond, are produced mainly in the heart and act as circulating hormones; however, CNP, which lacks the 'tail' sequence, functions as a paracrine or autocrine factor in the brain and peripheral tissues such as the endothelium (Chen \& Burnett 1998). Most biological actions of NPs are mediated by two distinct natriuretic peptide receptors (NPR-A and NPR-B), which are membrane guanylyl cyclases that generate cGMP as an intracellular second messenger. In mammals, ANP and BNP bind NPR-A with high affinity, whereas NPR-B is a selective receptor 
of CNP. This suggests that the NP family has diverged into two functionally and structurally distinct groups during vertebrate evolution. This is supported by genomic analysis because in human and mouse the ANP and BNP genes are localized in tandem on the same chromosome, while the GNP gene is present on a different chromosome (Ogawa et al. 1994, Tamura et al. 1996).

In elasmobranchs, however, GNP has been isolated from the heart and brain of dogfish, Triakis scyllia (Suzuki et al. 1994). Furthermore, CNP circulates in the blood at high concentrations (Suzuki et al. 1994), and vascularly perfused CNP stimulates $\mathrm{Cl}^{-}$secretion from the rectal gland of the dogfish, Squalus acanthias (Solomon et al. 1992, Silva et al. 1999), indicating that GNP functions as a circulating hormone in elasmobranchs. The major form of CNP stored in the heart and circulating in blood of Triakis is CNP prohormone (pro-GNP), but mature GNP (CNP-22) is stored in the brain as in all other species examined to date (Takei 2000). In order to confirm the absence of ANP, BNP and VNP in the dogfish heart, we removed the cloned GNP mRNA from the mRNA pool, because abundantly expressed CNP mRNA may hinder the amplification of other NP mRNAs that might be expressed in smaller amounts (Kawakoshi et al. 2001). However, the PGR cloning using primers that amplify any of the known NP cDNAs failed to detect other NPs except for a minor GNP cDNA with truncated 3'-untranslated sequences. Since CNP is present in all species thus far examined, and since CNP is structurally the most conserved peptide of the NP family, we hypothesized that GNP is the ancestral type of the NP family and that ANP, BNP and VNP appeared later by gene duplication. To verify this hypothesis, the identification of $\mathrm{NP}(\mathrm{s})$ in a phylogenetically more ancient vertebrate group is essential.

In the present study, we examined whether CNP is present in the heart and brain of the hagfish, Eptatretus burgeri, which belongs evolutionarily to the oldest vertebrate group, the cyclostomes. Previously, NP-like immunoreactivity has been detected in the brain and heart of Atlantic hagfish, Myxine glutinosa, using an antiserum raised against human ANP (Reinecke et al. 1987) and in the brain but not the heart of the same species using antisera raised against porcine BNP and CNP (Donald et al. 1992). We used the PGR-based method and degenerate primers that amplify the cDNAs of each type of NP and identified an mRNA encoding an NP precursor with a long C-terminal 'tail' sequence in the heart and brain of the hagfish; no other NP cDNAs including GNP were detected. Subsequently, the expression of this NP gene was determined in various tissues using PGR and the processed form in the heart, brain and plasma was examined by mass spectrometry in combination with affinity chromatography.

\section{Materials and Methods}

\section{Animals}

Hagfish, Eptatretus burgeri, of either sex that weighed $120 \pm 40 \mathrm{~g}(n=30)$ were collected from the Koajiro Bay near Misaki Marine Biological Station, University of Tokyo, in October 2000. The fish were anesthetized in $0.5 \%(\mathrm{w} / \mathrm{v}) 3$-aminobenzoic acid ethyl ester (Sigma) neutralized by sodium bicarbonate for $15 \mathrm{~min}$, and blood was collected from the caudal sinus into a chilled syringe containing $10 \mu \mathrm{l} / \mathrm{ml}$ blood of $10 \%$ 2K-EDTA. Tissues (brain, systemic heart, portal heart, intestine, gill and kidney) were then dissected out, quickly frozen in liquid nitrogen and kept at $-80{ }^{\circ} \mathrm{C}$ until use. The blood was centrifuged, and plasma was frozen in liquid nitrogen and stored at $-80{ }^{\circ} \mathrm{C}$ until use. All experiments described in this paper were performed in accordance with the Guidelines for Care and Use of Animals, approved by the University of Tokyo.

\section{Cloning of hagfish NP cDNA}

The cloning of the hagfish NP cDNA was achieved using $3^{\prime}$ - and 5'-RACE methodologies. Total RNA was extracted from the heart and brain using the AGPG method (Chomczynski \& Sacchi 1987). Poly- $\mathrm{A}^{+}$RNA was purified from the total RNA using Oligotex-dT30 (Takara, Kyoto, Japan) and $1 \mu \mathrm{g}$ was reverse transcribed to cDNA with a 3'-terminal adaptor using the 3'-Full RACE Core Set (Takara). PCR reactions were performed with one of the degenerate sense primers (see below) and the universal antisense adaptor primer, using a high-fidelity Ex-Taq DNA polymerase (Takara). The amplified products were ligated into a pT7 Blue T-vector (Novagen, Darmstadt, Germany), and the nucleotide sequences were determined using an automated DNA sequencer (PRISM 310; 
Perkin-Elmer/Applied Biosystems, Foster City, CA, USA). Five degenerate sense primers were designed that could amplify all known NP cDNAs: NP-1 (ANP and CNP), TGYTTYGGGGTNA ARGTGGAYMGNATHGG; NP-2 (ANP and BNP), GGCTGCTTTGGGSGNMGRATNGAY MGNAT; NP-3 (ANP and VNP), GGCTGTTTT GGTGMNMGRATKGANMGNAT; NP-4 (CNP only), GGCTGCTTTGGCYTNAARGTGGACM GNAT; and NP-5 (CNP only), CTGGACMGN ATCGGSKCGATGAGYGGNGT. The validity of these primers has been tested by amplification of NP cDNAs from the heart and brain of the eel, quail and dogfish. After an initial denaturation at $94{ }^{\circ} \mathrm{C}$ for $3 \mathrm{~min}, 40$ cycles of PGR were performed, each consisting of $1 \mathrm{~min}$ denaturation at $94{ }^{\circ} \mathrm{C}, 30 \mathrm{~s}$ annealing at varying temperatures, and $1.5 \mathrm{~min}$ extension at $72{ }^{\circ} \mathrm{C}$.

A circular or concatemeric first-strand cDNAmediated RACE method was applied to obtain the 5 '-end of the hagfish NP cDNA (Maruyama et al. 1995). One-half microgram poly- ${ }^{+}$RNA was reverse transcribed using a phosphorylated genespecific primer. After digestion of the RNA strand with RNase $\mathrm{H}$, the cDNA strand was circularized and/or joined into a concatemeric form by $\mathrm{T} 4$ RNA ligase. The resulting single-stranded cDNA was used as a template for amplification of the 5 '-end with gene-specific sense and antisense primers. PCR products were cloned and sequenced as described above. To confirm the nucleotide sequence obtained by the 5'- and 3'-RACE methods, specific sense and antisense primers were designed in the 5 '- and $3^{\prime}$-untranslated regions respectively, and the full length of the coding region was amplified, and subsequently cloned and sequenced as described above.

\section{Removal of Eptatretus burgeri NP mRNA with RNase H}

Since consensus sequences of all NPs are well conserved, it is almost impossible to design a specific primer that amplifies only a single-type of NP cDNA. In fact, all primers used in the current study are not specific for a single NP cDNA but cross-hybridize to varying degrees with multiple NP cDNAs at low annealing temperatures. Therefore, if an NP mRNA is expressed in large amounts in the heart or brain, this may prevent amplification of the other NP mRNAs that are expressed in smaller amounts. To address this potential problem, Eptatretus burgeri NP (EbuNP) mRNA was removed from the mRNA pool before cDNA synthesis (Kawakoshi et al. 2001). One microgram poly-A ${ }^{+}$RNA in $20 \mathrm{mM}$ Tris- $\mathrm{HCl}$ (pH 8.3), $50 \mathrm{mM} \mathrm{KCl}, 10 \mathrm{mM}$ dithiothreitol and $2.5 \mathrm{mM}$ $\mathrm{MgCl}_{2}$ were heat-denatured at $90{ }^{\circ} \mathrm{C}$ with $0.05 \mu \mathrm{g}$ oligonucleotide (melting temperature $63.9^{\circ} \mathrm{C}$ ) that is complementary to the $3^{\prime}$-untranslated region of the cloned EbuNP mRNA (nucleotides 12161235). The mixture was cooled gradually to an annealing temperature of $70{ }^{\circ} \mathrm{C}$. RNase $\mathrm{H}(0.02$ units) was then added and the reaction was incubated at $70{ }^{\circ} \mathrm{C}$ for $20 \mathrm{~min}$. After purification with phenol/chloroform, 3'-RACE was performed as described above. To confirm the specific removal of the cloned EbuNP mRNA, hagfish $\beta$-actin and vasotocin cDNAs (Suzuki et al. 1995) were amplified in parallel with the EbuNP cDNA.

\section{Tissue expression of EbuNP mRNA}

The expression of the EbuNP gene was studied in the cardiac atrium, ventricle, portal heart, brain, gill, kidney and intestine from three individuals. One microgram total RNA from each tissue was reverse-transcribed using the Superscript FirstStrand Synthesis System for RT-PCR (Invitrogen), and one-fortieth of the first-strand cDNA was subjected to PCR amplification with specific sense and antisense primers (nucleotides 344-363 and 672-691 of EbuNP cDNA). After an initial denaturation at $94^{\circ} \mathrm{C}$ for $3 \mathrm{~min}, 30$ cycles of PCR were performed, each consisting of 1 min denaturation at $94{ }^{\circ} \mathrm{C}, 30 \mathrm{~s}$ annealing at $60{ }^{\circ} \mathrm{C}$ and $1.5 \mathrm{~min}$ extension at $72{ }^{\circ} \mathrm{C}$. The resulting PCR products were electrophoresed through a $1.2 \%$ (w/v) agarose gel and were detected with an automatic image analyzer (FLA 2000; Fuji Film) after staining with ethidium bromide. As a reference control, hagfish $\beta$-actin mRNA was amplified under the same conditions.

\section{Preparation of affinity column}

An anti-EbuNP serum was generated for preparation of the affinity column. As an antigen, a 30 amino acid peptide at the C-terminus of the EbuNP precursor was synthesized in a peptide synthesizer (Model 430A; Applied Biosystems). A disulfide bond was formed at the two Cys residues. To enable future iodination, a Tyr residue was 
added to the N-terminus of the synthetic EbuNP. It is generally known that addition or deletion of the N-terminal amino acids scarcely influences the biological activity of NPs (Takei 2000). The antiserum was raised in two rabbits against the synthetic EbuNP conjugated to BSA with glutaraldehyde. The antibody titer was examined by ELISA every month. The peptide synthesis and antibody production were done at the Peptide Institute Inc., Osaka, Japan. The IgG was purified from $2 \mathrm{ml}$ antiserum using GammaBind Plus Sepharose (Amersham Pharmacia Biotech). The affinity column was prepared using an Affi-Gel $\mathrm{Hz}$ Immunoaffinity Kit (Bio-Rad, Richmond, CA, USA) by coupling the purified IgG with Affi-Gel $\mathrm{Hz}$ hydrazide gel. Before sample application, $10 \mathrm{nmol}$ BSA, which was used as a carrier for IgG production, was dissolved in $10 \mathrm{ml}$ application buffer $(0 \cdot 01 \mathrm{M}$ PBS, pH 7.2) and was applied onto the column to prevent recovery of components that bind to anti-BSA IgG.

\section{Sample preparation from tissues and plasma}

The extracts of the hagfish heart and brain were prepared based on the method of Takei et al. (1989) for isolation of eel ANP. The tissues (systemic hearts and brains, $\sim 3 \mathrm{~g}$ each) were heated in $10 \mathrm{ml}$ distilled water in a boiling water bath for $5 \mathrm{~min}$, and rapidly cooled to $4{ }^{\circ} \mathrm{C}$. Acetic acid was then added to a final concentration of $1 \mathrm{M}$, and the tissues were homogenized in a Polytron homogenizer for $1 \mathrm{~min}$ at the maximum speed. After centrifugation at $25000 \boldsymbol{g}$ for $30 \mathrm{~min}$ at $4{ }^{\circ} \mathrm{C}, 20 \mathrm{ml}$ acetone were added to the supernatant. After $1 \mathrm{~h}$ at $4{ }^{\circ} \mathrm{C}$, the mixture was centrifuged and the supernatant was lyophilized. The dried materials were dissolved in $3 \mathrm{ml} 1 \mathrm{M}$ acetic acid, to which $300 \mathrm{ml}$ cold acetone were added and kept at $4{ }^{\circ} \mathrm{C}$ for more than $3 \mathrm{~h}$. Hagfish plasma $(100 \mathrm{ml})$ was treated as above without Polytron homogenization. Finally, the $99 \%$ acetone mixture was centrifuged at $15000 \boldsymbol{g}$ for $30 \mathrm{~min}$ at $4{ }^{\circ} \mathrm{C}$, and the precipitate was dissolved in coupling buffer (heart and brain, $5 \mathrm{ml}$; plasma, $20 \mathrm{ml}$ ) for affinity chromatography. Synthetic $\left[\mathrm{Tyr}^{0}\right]$-EbuNP $(20 \mathrm{pmol})$, which is not an endogenous peptide, was added to each extract to examine the performance of the affinity column. Bound materials were eluted with $3 \mathrm{ml} 0 \cdot 1 \mathrm{M}$ acetic acid and were subjected to mass spectrometry as described below.

\section{Mass spectrometry}

The affinity-purified samples of plasma, heart and brain were subjected to mass spectrometry in a Q-Tof mass spectrometer (Micromass, Manchester, UK) combined with capillary HPLC. This mass spectrometry allows the determination of the molecular mass from semi-purified samples after separation by reverse-phase HPLC with an accuracy of $< \pm 0.5 \mathrm{Da}$. The detailed setup and usage of the mass spectrometer have been described previously (Yasuda-Kamatani \& Yasuda 1999).

\section{Molecular phylogenetic analysis}

Deduced amino acid sequence of EbuNP prohormone was aligned with those of ANP, BNP, VNP and CNP of other species by the T-Coffee program (Notredame et al. 2000). The aligned sequences were then analyzed by the ClustalW program with the default settings except that TOSSGAPS was turned off.

\section{Results}

\section{Cloning of cDNAs encoding EbuNP}

An NP cDNA was cloned from the heart and brain of hagfish by NP-1 and NP-2 primers that aim to amplify ANP and BNP cDNA, but not by NP-3, NP-4 and NP-5 (Fig. 1). No other NP cDNAs were amplified from the heart and brain using any of the primers. Thus, we named the NP of Eptatretus burgeri EbuNP. The EbuNP precursor deduced from the cDNA sequence was composed of 162 amino acid residues carrying a mature peptide at the G-terminus (Fig. 1). The N-terminal 23 amino acid peptide of the precursor was predicted to be a signal peptide (Nielsen et al. 1999). The C-terminal portion had two Cys residues that may form a ring structure flanked by a disulfide bond. The C-terminal 'tail' sequence of ten amino acid residues extended from the ring structure. The unique Arg residue existed just after the second Gys. The Gly residue at the $\mathrm{C}$-terminus may be an amidation signal. The analysis of three clones from the heart and brain showed that the nucleotide sequence of EbuNP cDNA was identical in the heart and brain even in the untranslated regions. 


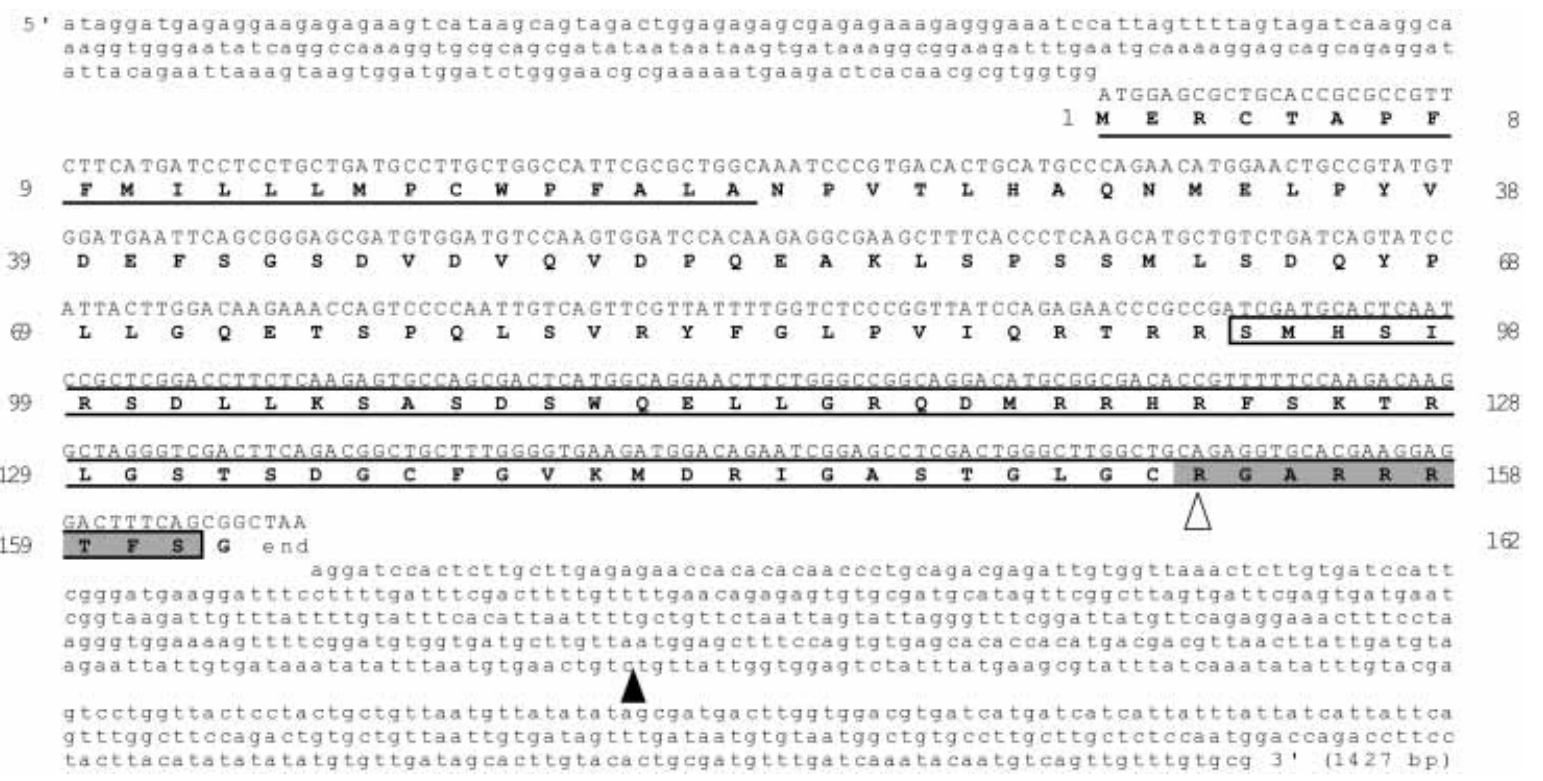

Figure 1 Nucleotide sequence of cloned cDNA encoding the EbuNP precursor. The amino acid sequence of prepro-EbuNP deduced from the nucleotide sequence is also shown. The numbers indicate the position of amino acid residues in the precursors and the inferred signal peptide is underlined. The processed form in the heart and plasma is boxed. The open arrowhead indicates Arg just after the intramolecular ring, and subsequent tail residues are shaded. Mass spectrometry of the processed sequence showed that the C-terminal Gly was used for amidation of the Ser residue. The closed arrowhead indicates the polyadenylation site of shorter cDNA obtained after RNase $\mathrm{H}$ treatment (see text and Fig. 2b). The EbuNP cDNA sequence has been deposited in the DDBJ database with an accession number of AB087732.

\section{RNase H digestion}

The RNase $\mathrm{H}$ treatment of the heart and brain mRNA after hybridization with the oligonucleotide at the $3^{\prime}$-untranslated region abolished the amplification of the EbuNP cDNA, which should have appeared at $\sim 800$ bp by RT-PCR (Fig. 2a). In contrast, $\beta$-actin and vasotocin $\mathrm{cDNAs}$ were amplified after the enzyme treatment, showing the specific removal of the EbuNP mRNA. After removal of the EbuNP, further amplification by 3'-RACE produced a smaller, faint band (Fig. 2b). Sequence analysis revealed that this cDNA encodes the same EbuNP precursor with a truncated $3^{\prime}$-untranslated sequence, but not that of different NP species (Fig. 1).

\section{Tissue distribution of EbuNP gene expression}

RT-PGR showed that EbuNP mRNA was expressed abundantly in the systemic heart (atrium and ventricle) and brain of hagfish (Fig. 3). In addition to the systemic heart, strong expression was detected in the portal heart that is unique to the hagfish circulatory system. A low level of expression was also observed in the intestine, but no signal was detected in the gill and kidney (Fig. 3).

\section{Molecular forms of EbuNP in plasma and tissues}

Multiple charged ions were observed at $\mathrm{m} / \mathrm{z}$ $847 \cdot 29,953 \cdot 04,1089 \cdot 06,1270 \cdot 44$ and $1524 \cdot 39$ in the HPLC-mass spectrometry analysis with the affinity-purified heart and plasma samples (Fig. 4), which were deconvoluted to $7616 \cdot 6$ Da. This value was in good agreement with the theoretical value $(7616 \cdot 6)$ of one from the possible processed sequence (from $\mathrm{Ser}^{94}$ to $\mathrm{Ser}^{161}$ ) with a disulfide bond and an amidated C-terminus (Fig. 1). The N-terminal processing site $\left(\mathrm{Arg}^{90}{ }^{-\mathrm{Thr}^{91}} \mathrm{Arg}^{92}{ }_{-}\right.$ $\mathrm{Arg}^{93}$ ) corresponded to the processing motif of a major processing enzyme, furin (Hosaka et al. 1991). Gly ${ }^{162}$ at the C-terminus was used for amidation of Ser ${ }^{161}$. Since the confidence limit of the mass spectrometry used in this study was within $\pm 0.5 \mathrm{Da}$, the amidation of the G-terminal 


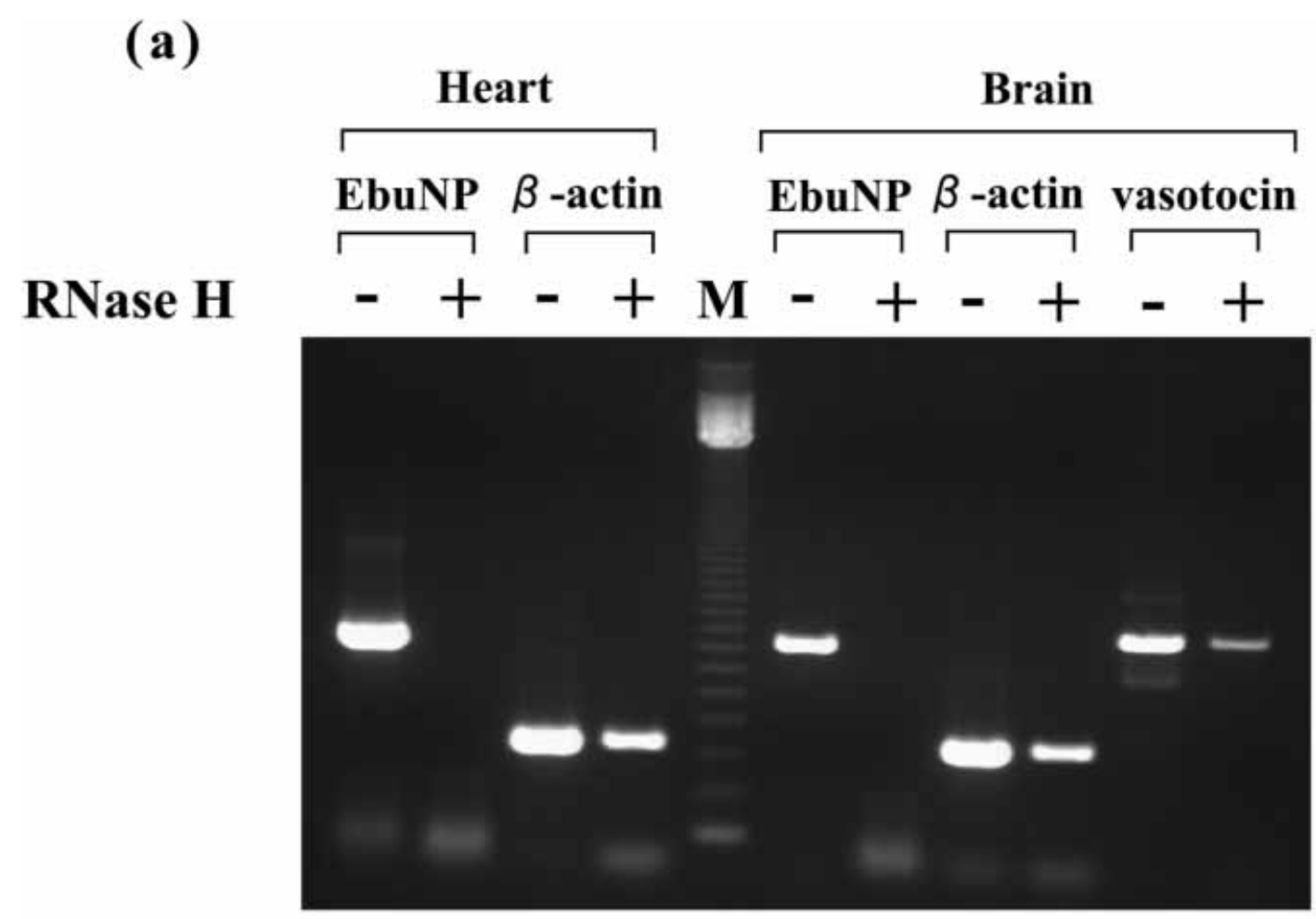

(b)
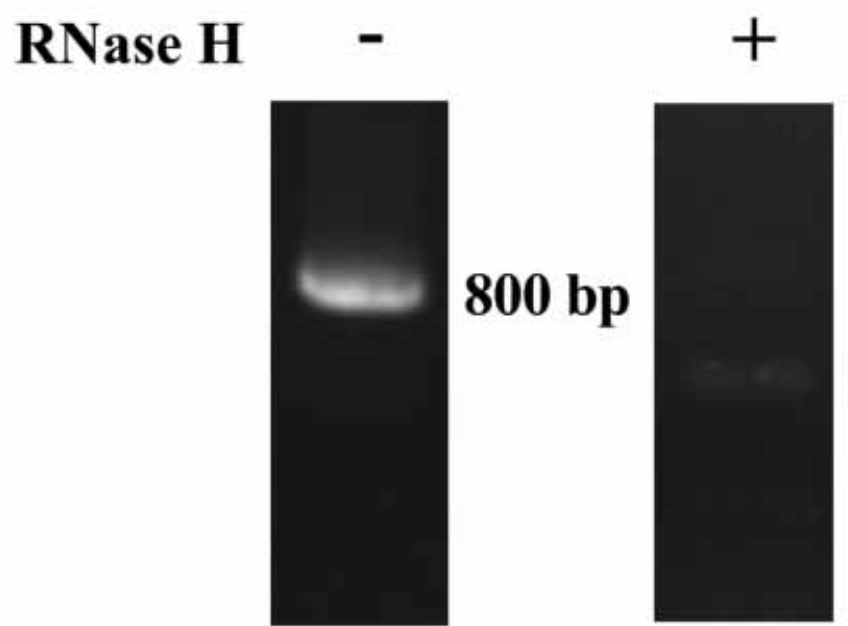

500 bp

Figure 2 Effect of specific digestion of EbuNP mRNA with RNase H. (a) RT-PCR of poly-A $\mathrm{A}^{+} \mathrm{RNA}$ samples from systemic heart and brain with $(+)$ or without $(-)$ RNase $\mathrm{H}$ treatment. Amplification of EbuNP cDNA was completely abolished while $\beta$-actin and vasotocin were still amplified after treatment. (b) 3'-RACE of intact (-) and RNase $\mathrm{H}$-treated (+) poly- $\mathrm{A}^{+} \mathrm{RNA}$ of systemic heart. The strong band of cloned EbuNP at 800 bp disappeared but a new faint band appeared at $500 \mathrm{bp}$, which was a truncated form of EbuNP CDNA (for details, see text). M, 123 bp molecular marker. 

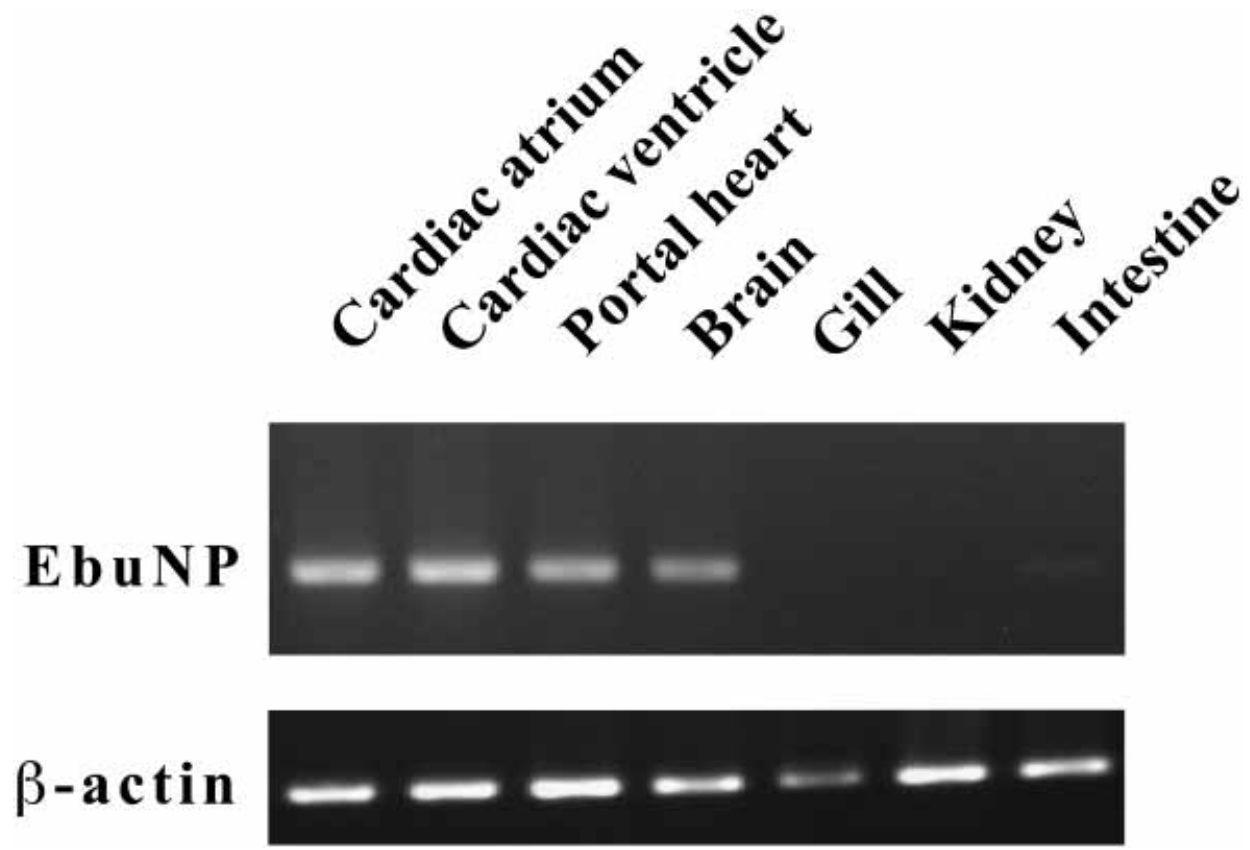

Figure 3 Distribution of EbuNP mRNA in various hagfish tissues as determined by RT-PCR with $25 \mathrm{ng}$ total RNA and 30 cycles of PCR. Hagfish $\beta$-actin was used as reference controls. Similar results were obtained in three individuals.

Ser was evident. Since the peak for the $4+$ charge state ion of synthetic EbuNP (molecular mass $=3280 \cdot 6)$ was detected in the mass spectrometry (Fig. 4b), the performance of the affinity chromatography was confirmed. From the affinitypurified brain samples, no distinct peaks corresponding to the possible processed sequences, including those of the truncated C-terminal 'tail' sequence, were obtained.

\section{Molecular phylogenetic analysis}

The alignment of NP prohormones from various species shows that EbuNP has low similarity to ANP, BNP, VNP and CNP in the prosegment (Fig. 5). EbuNP cannot be categorized into any NPs even by comparison with mature sequences. The molecular phylogenetic tree depicted with prohormone sequences also showed that EbuNP does not belong to any group of NPs (Fig. 6).

\section{Discussion}

The hagfish is evolutionarily the oldest vertebrate species that diverged over 550 million years ago

www.endocrinology.org from the other vertebrate species processed (Sower \& Kawauchi 2001). It seems that their genes coding for proteins may have been modified profoundly during the long history of evolution. To our knowledge, only three hormone genes have been cloned from the hagfish: insulin (Chan et al. 1981), insulin-like growth factor (Nagamatsu et al. 1991) and vasotocin (Heierhorst et al. 1992, Suzuki et al. 1995), all of which are well-conserved across different classes of vertebrates. In the present study, an NP cDNA was successfully cloned from the heart and brain of hagfish. No other NP cDNA was cloned even when PCR primers that can amplify all members of the NP family were used. No other type of NP was obtained even after specific removal of the cloned EbuNP cDNA; only a minor EbuNP cDNA with a partially truncated 3 '-untranslated region was amplified. Therefore, it is highly probable that EbuNP is the sole NP in this evolutionarily old vertebrate species. The EbuNP precursor was not categorized into any group of ANP, BNP, VNP or CNP (Fig. 6). Therefore, we regard it as a novel type.

We previously reported that only GNP is present in elasmobranchs (Kawakoshi et al. 2001), although 


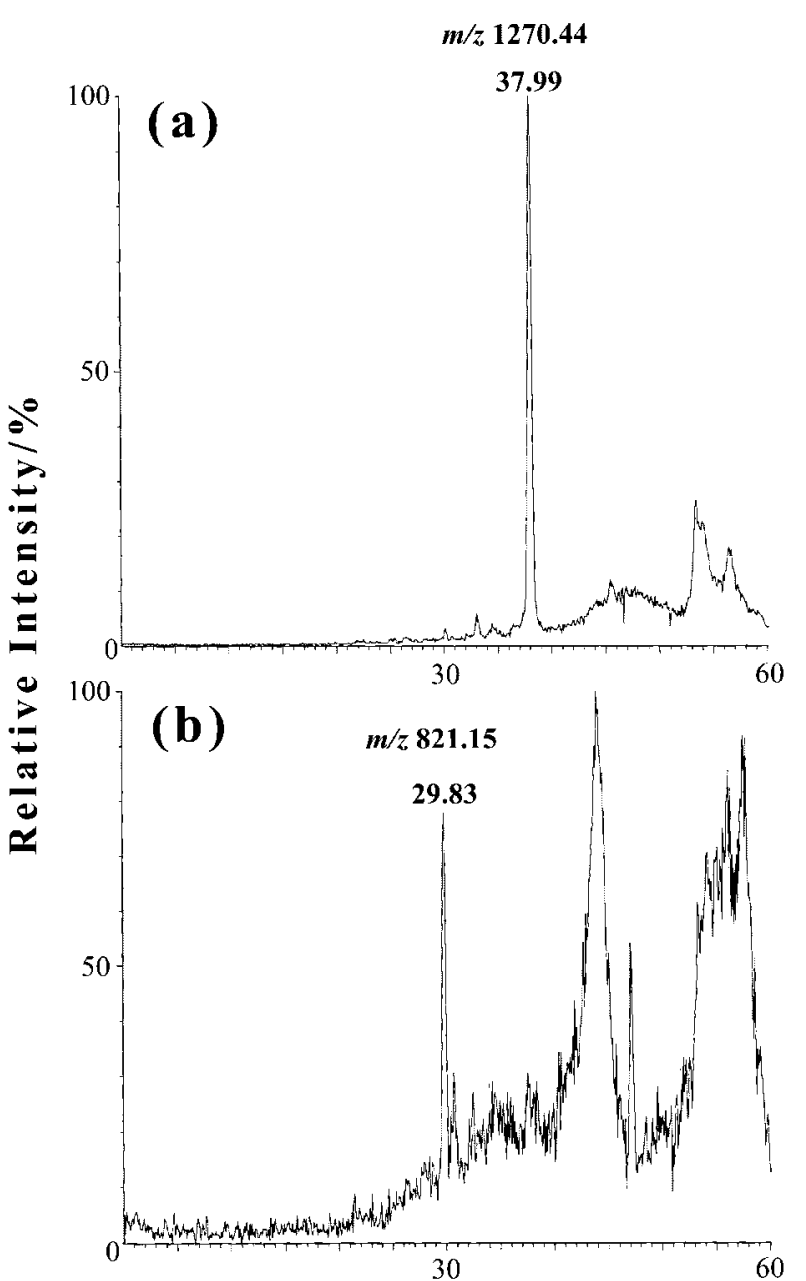

Time/min

Figure 4 Selected ion chromatograms in capillary reversed-phase HPLC/Q-Tof mass spectrometry analysis with an affinity-purified sample from the hagfish heart. (a) A peak at 37.99 min corresponding to the 6+ charge state of the processed EbuNP (molecular mass $=7616 \cdot 6$ ). (b) A peak at 29.83 min corresponding to the $4+$ charge state of synthetic $\left[\mathrm{Tyr}^{\circ}\right]$-EbuNP (molecular mass $=3280 \cdot 6$ ). Similar peaks were also observed in the plasma sample at the elution positions of the processed EbuNP and synthetic [Tyr ${ }^{\circ}$-EbuNP (data not shown). The processed form predicted from the molecular mass is shown in Fig. 1.

at least three different NPs have been identified in teleost fish, amphibians and mammals. Therefore, it is most likely that GNP is the ancestral type of the NP family, and other NPs have evolved by gene duplication during the transition from cartilaginous fish to bony fish. However, it is apparent that the novel EbuNP identified in the current study is not
CNP because of the presence of a long C-terminal tail sequence. In the tail sequence, three consecutive basic amino acid residues (Arg-Arg-Arg) are present, a characteristic shared with eel and trout VNP (Takei 2000). The tail sequence is amidated at the C-terminus, which is a characteristic shared only with eel ANP (Takei et al. 1997). On the other hand, in the prosegments of ANP, BNP and VNP, a highly conserved region is present (Fig. 5), which is not observed in the prosegment of EbuNP and CNP. This conserved sequence has been suggested to form a motif for transcriptional regulation (Seidler et al. 1999). In terms of structural characteristics, therefore, it is not possible to classify EbuNP to any of the known members of the NP family.

Only a single peptide that corresponded to the appropriate molecular mass of the processed EbuNP was recovered from the heart and plasma after affinity chromatography using antisera raised against the putative C-terminal mature peptide. The pro-EbuNP that should be present in the heart was not recovered from the affinity chromatography, probably because of its low affinity to the antiserum. Judging from the molecular mass, the processed form in the heart and plasma consisted of 68 amino acid residues with an amidated C-terminus. The processing signal of the precursor (Arg-X-Arg-Arg) showed that the peptide is most probably cleaved by furin, a member of the Kex2 family of major processing enzymes (Hosaka et al. 1991). In the NP family, only BNP is known to be processed by furin, and a processed form secreted from the heart circulates in the blood of mammals (Tateyama et al. 1990, Togashi et al. 1991). Since mature ANP, VNP and CNP are processed by enzymes other than furin, EbuNP is similar to BNP in this respect.

The EbuNP gene was expressed in the portal heart in addition to the atrium and ventricle of the systemic heart. The portal heart is an organ unique to hagfish that helps pump a large volume of blood under low arterial pressure (Forster 1998). Previously, Reinecke et al. (1987) reported that secretory granules are present in both the systemic and portal heart of Myxine glutinosa which contain immunoreactive ANP. Thus the present result is consistent with their observation. In addition to cardiac tissues, EbuNP was expressed in small amounts in the intestine. In mammals and teleost fish, ANP is produced in the intestine and regulates 
EbuNP

human ANP porcine ANP Xenopus ANP eel ANP

human BNP porcine BNP

chicken BNP

Xenopus BNP eel VNP

trout VNP human CNP porcine CNP eel CNP dogfish CNP
$----N P V T L H A Q N M E L--P Y V D E F$

- NPVYGVSNADT-MDFYNE --- SPAY S SVL S SDL - T D L KN I LE - R L EDRV- T TEE P MAP SQDL- - F AONY D --- HVLSRPYSANDL- -ARLKSFLE-QFEDTL--AREEVADNPGDY - EDAKPE $---H P L G S P G S A S D L E T S G L Q E Q R N-H L Q G K L--S E L Q V E Q T S---L E P L Q E S P$ - - HPLGGAGLASEL--PG IQELLD-RLRDRV--SELQAERTD---LEPLRQDR $----\mathbb{N} P I Y N L S$ PAKEL- - ASMEA A LE - R LEDKF- - AL I E ALE S N P DLQE PQTQEE $---H P L M D L D K D R D L--D T F K G V L E-R L E E K L--A L I E A L D A--D A T E P K S Q D-$ $----\mathbb{N} P L F N R N N V Q E L--E N L K D L I H-Q L E E R V--A V N E E---P E V Y--P E S E D M$ ---- TPYASRN - VQEL--ENLKDLIQ-RLEDKL- - TVNEE-- -NYAY - - PSESED KPGAPPKVPRTP KPGAPPKVPRTP - - DSRALRT PVDA I - Q Q FVE QFLE - HYNDLL - N I D D LENQ - - - TGDQLE -

EbuNP

human ANP porcine ANP Xenopus ANP eel ANP

human BNP porcine $B N P$ chicken BNP Xenopus BNP eel VNP

trout VNP human CNP porcine CNP eel CNP dogfish CNP
70 AGAALSPLPEVPPW- TGEVSPAQRDGGALGRGPWDSSDRSAL 110 69 VGAPLSPLLEVPPW 68 AADSSNS---APSW-67 EAERGQP---GPEW--- $--\cdots$ 72 RP - - TGVWK--

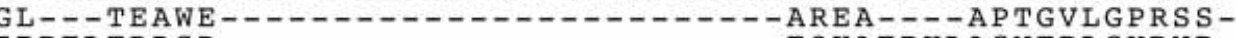
I PPELTDDSD-. VDAARLPVID KMDAEEEDAG I S P-.--

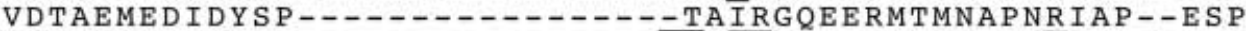
E PQAA G G G KKGDKAPG G - - GANLKGDRSRLLRDLRVDTKSRAAWARLIQE-H E PQAAG G G Q K K GDKTPG G G---GANLKGDRSRLLRDLRVDTKSRA AWARLLHE - H SPOPP L S GLKVIAE Y P - - EFNADQS DLELPW--.-.-.-.-- DRESREIGGR-PFRQEAVLARLLKDLS

99
93
90
99
96
99
98
92
92
95
102

B

EbuNP 117 QDMR-RHRFSKTRLGSTSDGCFGVKMDRIGASTGLGCRGARR----RTFS-NH2

Figure 5 Comparison of amino acid sequences of EbuNP and other NP prohormones. The amino acid residues identical to EbuNP are highlighted in black. Conserved residues in ANP, BNP and VNP are shaded in box A. Box B shows 'tail' sequences and the disulfide bond is combined by a solid line. The numbers indicate the position of amino acid residues in the precursors. Accession numbers: human ANP, NM-006172; porcine ANP, X54669; Xenopus ANP, AF287050; eel ANP, AB019372; human BNP, M25296; porcine BNP, M25547; chicken BNP, X57702; Xenopus BNP, BG346231; eel VNP, AB019371; trout VNP, AB076604; human CNP, NM-024409; porcine CNP, M64758; eel CNP, D88022; dogfish CNP, AB047081.

$\mathrm{NaCl}$ absorption in a paracrine or autocrine fashion (Loretz et al. 1997, Gonzalez Bosc et al. 2000). ANP is also produced in the kidney as urodilatin in mammals (Meyer et al. 1998) and teleost fish (Takei et al. 1997), but no expression of EbuNP was detected in the hagfish kidney. 


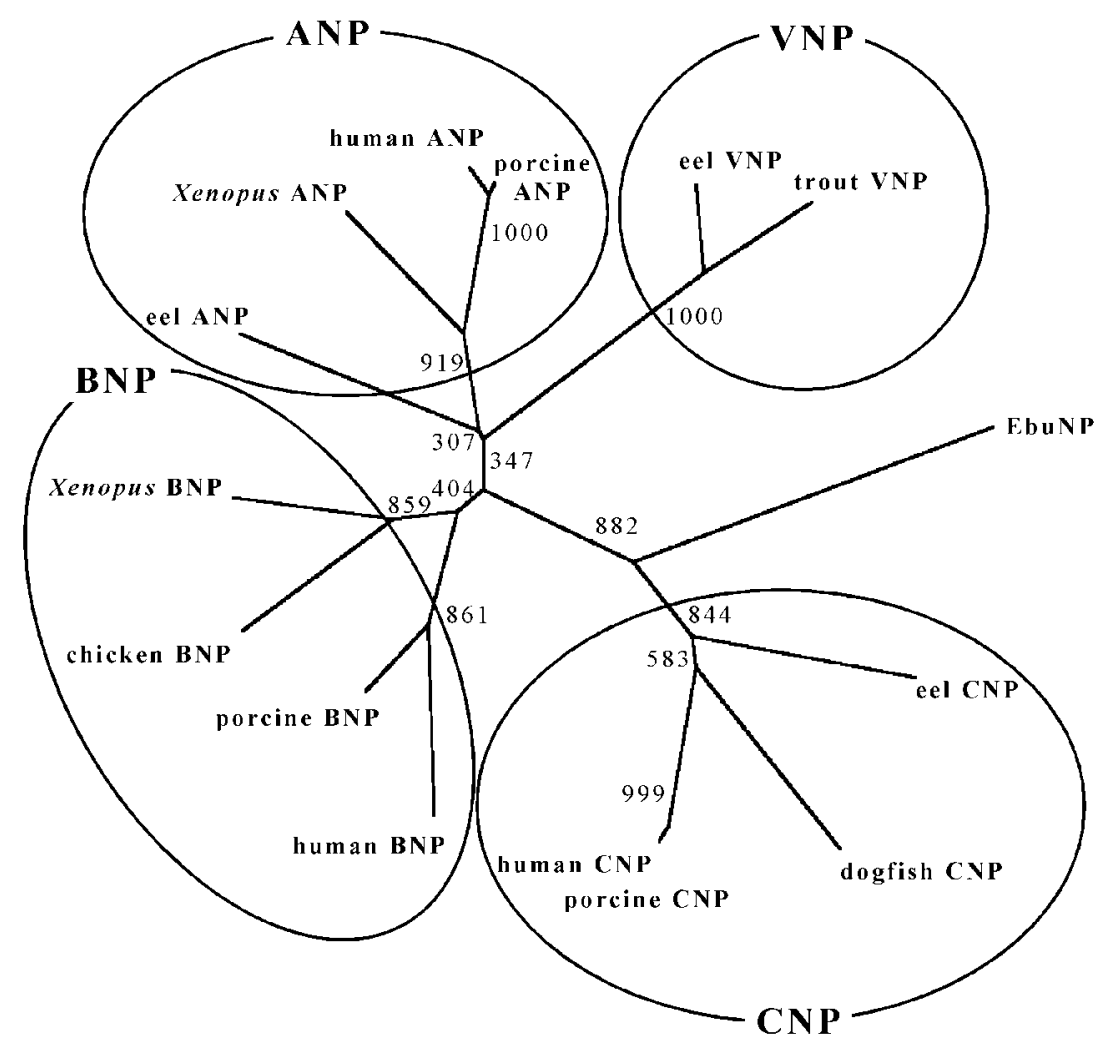

Figure 6 Molecular phylogenetic analysis of NP family precursors. Aligned sequences as shown in Fig. 5 were analyzed by the ClustalW program. ANP, BNP, VNP and CNP precursors are separated into distinct groups, while EbuNP does not belong to any group of known NPs. Bootstrap analysis is performed with 1000 repetitions.

Together with the absence of EbuNP expression in the gill, the peptide may not act as a local paracrine factor in the osmoregulatory organs of the hagfish.

Since only EbuNP gene is expressed in the brain, the hagfish is the only species in which GNP is not detected in the brain. Reinecke et al. (1987) reported the presence of immunoreactive ANP in the hagfish brain, which coincides with the abundant expression of EbuNP in the brain found in this study. In spite of the abundant expression as detected by RT-PCR, no immunoreactive EbuNP could be recovered from the brain after affinity chromatography using the antiserum directed to the C-terminal portion of the precursor. The Arg residue of EbuNP located just after the intramolecular ring, which is unique to $\operatorname{EbuNP}$ (Fig. 5), may serve as a processing signal in the brain just as in ANP-(4-28), which is processed after a single Arg residue in the mammalian brain. The cleavage of the 'tail' sequence and subsequent removal of the
Arg residue by a carboxypeptidase may produce a GNP-like peptide in the brain. The long C-terminal 'tail' sequence of EbuNP is unique to this molecule and strongly hydrophilic, so that this portion may be highly immunogenic in the rabbit. Thus, the failure to recover EbuNP from the brain after affinity chromatography could be accounted for by the lack of the 'tail' sequence. The fact that immunoreactive GNP is present in the brain but absent from the heart of Atlantic hagfish supports this idea (Donald et al. 1992). In elasmobranchs that have only GNP in the heart and brain, GNP-22 is stored in the brain as in other vertebrate species, while pro-GNP is stored in the heart and circulates in the blood (Suzuki et al. 1994). In mammals, ANP-(4-28) and -(5-28) are stored in the brain (Ueda et al. 1987), while pro-ANP is stored in the heart. ANP-(1-28) is cleaved off from pro-ANP by the enzyme corin, at the time of secretion and circulates in the blood (Wu et al. 2002). Thus, the 
processing of NP family members generally differs between the endocrine heart and paracrine brain.

The identification of a novel NP that is equally similar to ANP, BNP, VNP and CNP in evolutionarily the oldest vertebrate species makes the molecular evolution of the NP family more complicated. One possibility for the evolution is that CNP is the ancestral type as found in elasmobranchs, but a mutation occurred within the hagfish lineage in the course of evolution. Another possibility is that EbuNP is the ancestral type. In this case, EbuNP has changed into GNP during the course of evolution to cartilaginous fishes. This occurs by one point mutation of the Arg residue just after the intramolecular ring that changes Arg to a stop codon. The mutation may have occurred before genome duplication before evolution to gnathostomes as hypothesized by Escriva et al. (2002) because our preliminary data showed that GNP is present in the brain and heart of lamprey, Lampetra japonica (A Kawakoshi, S Hyodo \& Y Takei, unpublished observations). Since dogfish expresses only one CNP gene (Kawakoshi et al. 2001), one of the duplicated CNP genes may have been lost during the course of evolution. Since only one NP is present in the hagfish, it is intriguing to examine which type(s) of NP receptors are present in the hagfish. In mammals and teleost fishes, it has been demonstrated that NPR-A is a receptor for ANP, BNP and VNP, and NPR-B is a specific receptor for GNP. In elasmobranchs where only GNP exists, only NPR-B has been identified (Aller et al. 1999). In the hagfish, a partial sequence of an NP receptor has been cloned (Callahan et al. 2000), but it is too short to determine the receptor type. Identification of more NP ligands and NP receptors in species at various phylogenetic positions will enable us to show a more clear picture of the evolution of the NP system in vertebrates.

\section{Acknowledgements}

The authors thank Dr John A Donald of Deakin University for critical reading of this manuscript and to Dr Nobuo Suzuki of Kanazawa University for his help in sampling hagfish tissues. We are also grateful to the staff of Misaki Marine Biological Station, the University of Tokyo, for collection of hagfish. This work was supported in part by Grant-in-Aid for Creative Basic Research
(12NP0201) from the Ministry of Education, Culture, Sports, Science and Technology of Japan, and for Scientific Research (13304063) from the Japan Society for the Promotion of Science to Y T.

\section{References}

Aller SG, Lombardo ID, Bhanot S \& Forrest JN Jr 1999 Cloning, characterization, and functional expression of a CNP receptor regulating CFTR in the shark rectal gland. American fournal of Physiology 276 C442-C449.

Brenner BM, Ballermann BJ, Gunning ME \& Zeidel ML 1990 Diverse biological actions of atrial natriuretic peptide. Physiological Reviewes 70 665-699.

Callahan W, Forster M \& Toop T 2000 Evidence of a guanylyl cyclase natriuretic peptide receptor in the gills of the New Zealand hagfish Eptatretus cirrhatus (Class Agnatha). Fournal of Experimental Biology 203 2519-2528.

Chan SJ, Edmin SO, Kwok SC, Kramer JM, Falkmer S \& Steiner DF 1981 Messenger RNA sequence and primary structure of preproinsulin in a primitive vertebrate, the Atlantic hagfish. Fournal of Biological Chemistry 256 7595-7602.

Chen HH \& Burnett JC Jr 1998 C-type natriuretic peptide: the endothelial component of the natriuretic peptide system. Fournal of Cardiovascular Pharmacology 32 S22-S28.

Chomczynski P \& Sacchi N 1987 Single-step method of RNA isolation by acid guanidinium thiocyanate-phenol-chloroform extraction. Analytical Biochemistry 162 156-159.

Donald JA, Vomachka AJ \& Evans DH 1992 Immunohistochemical localisation of natriuretic peptides in the brains and hearts of the spiny dogfish Squalus acanthias and the Atlantic hagfish Myxine glutinosa. Cell and Tissue Research 270 535-545.

Escriva H, Manzon L, Youson J \& Laudet V 2002 Analysis of lamprey and hagfish genes reveals a complex history of gene duplications during early vertebrate evolution. Molecular Biology and Evolution 19 1440-1450.

Evans DH 1990 An emerging role for a cardiac peptide hormone in fish osmoregulation. Annual Review of Physiology 52 43-60.

Farrell AP \& Olson KR 2000 Cardiac natriuretic peptides: a physiological lineage of cardioprotective hormone? Physiological and Biochemical Zoology 73 1-11.

Forster ME 1998 Cardiovascular function in hagfishes. Blood propulsors. In The Biology of Hagfishes, edn 1, pp 240-245. Eds JM Jorgensen, JP Lomholt, RE Weber \& H Malte. London; New York: Chapman \& Hall Co.

Gonzalez Bosc LV, Majowicz MP \& Vidal NA 2000 Effects of atrial natriuretic peptide in the gut. Peptides 21 875-887.

Heierhorst J, Lederis K \& Richter D 1992 Presence of a member of the Tcl-like transposon family from nematodes and Drosophila within the vasotocin gene of a primitive vertebrate, the Pacific hagfish Eptatretus stouti. PNAS $896798-6802$.

Hosaka M, Nagahama M, Kim W, Watanabe T, Hatsuzawa K, Ikemizu J, Murakami K \& Nakayama K 1991

Arg-X-Lys/Arg-Arg motif as a signal for precursor cleavage catalyzed by furin within the constitutive secretory pathway. Fournal of Biological Chemistry 266 12127-12130.

Kawakoshi A, Hyodo S \& Takei Y 2001 CNP is only natriuretic peptide in an elasmobranch fish, Triakis scyllia. Zoological Science $\mathbf{1 8}$ 861-868.

Loretz CA \& Pollina C 2000 Natriuretic peptides in fish physiology. Comparative Biochemistry and Physiology 125 169-187.

Loretz CA, Pollina C, Kaiya H, Sakaguchi H \& Takei Y 1997 Local synthesis of natriuretic peptides in the eel intestine. Biochemical and Biophysical Research Communications 238 817-822. 
Maruyama IN, Rakow TL \& Maruyama HI 1995 cRACE: a simple method for identification of the $5^{\prime}$ end of mRNAs. Nucleic Acids Research 23 3796-3797.

Meyer M, Richter R \& Forssmann WG 1998 Urodilatin, a natriuretic peptide with clinical implications. European Fournal of Medical Research 3 103-110.

Nagamatsu S, Chan SJ, Falkmer S \& Steiner DF 1991 Evolution of the insulin gene superfamily. Sequence of a preproinsulin-like growth factor cDNA from the Atlantic hagfish. Fournal of Biological Chemistry 266 2397-2402.

Nielsen H, Brunak S \& von Heijne G 1999 Molecular learning approaches to the prediction of signal peptides and other protein sorting signals. Protein Engineering 12 3-9.

Notredame C, Higgins D \& Heringa J 2000 T-Coffee: a novel method for multiple sequence alignments. Fournal of Molecular Biology 302 205-217.

Ogawa Y, Itoh H, Yoshitake Y, Inoue M, Yoshimasa T, Serikawa T \& Nakao K 1994 Molecular cloning and chromosomal assignment of the mouse $\mathrm{C}$-type natriuretic peptide (CNP) gene (Nppc): comparison with the human CNP gene (NPPC). Genomics 24 383-387.

Reinecke M, Betzler D \& Forssmann WG 1987 Immunohistochemistry of cardiac polypeptide hormones (cardiodilatin/atrial natriuretic polypeptide) in brain and hearts of Myxine glutinosa (Cyclostomata). Histochemistry 86 233-239.

Seidler T, Pemberton C, Yandle T, Espiner E, Nicholls G \& Richards M 1999 The amino terminal regions of proBNP and proANP oligomerise through leucine zipper-like coiled-coil motifs. Biochemical and Biophysical Research Communications 255 495-501.

Silva P, Solomon RJ \& Epstein FH 1999 Mode of activation of salt secretion by $\mathrm{C}$-type natriuretic peptide in the shark rectal gland. American Fournal of Physiology 277 R1725-R1732.

Solomon R, Protter A, Mcenroe G, Porter G \& Silva P 1992 C-type natriuretic peptides stimulate chloride secretion in the rectal gland of Squalus acanthias. American Fournal of Physiology 262 R707-R711.

Sower SA \& Kawauchi H 2001 Update: brain and pituitary hormones of lampreys. Comparative Biochemistry and Physiology 129 291-302.

Suzuki M, Kubokawa K, Nagasawa H \& Urano A 1995 Sequence analysis of vasotocin cDNAs of the lamprey, Lampetra japonica, and the hagfish, Eptatretus burgeri: evolution of cyclostome vasotocin precursors. Fournal of Molecular Endocrinology 14 67-77.
Suzuki R, Togashi K, Ando K \& Takei Y 1994 Distribution and molecular forms of C-type natriuretic peptide in plasma and tissue of a dogfish, Triakis scyllia. General and Comparative Endocrinology 96 378-384.

Takei Y 2000 Structural and functional evolution of the natriuretic peptide system in vertebrates. International Review of Cytology 194 $1-66$.

Takei Y, Takahashi A, Watanabe TX, Nakajima K \& Sakakibara S 1989 Amino acid sequence and relative biological activity of eel atrial natriuretic peptide. Biochemical and Biophysical Research Communications 164 537-543.

Takei Y, Ueki M, Takahashi A \& Nishizawa T 1997 Cloning, sequencing, tissue-specific expression, and prohormone isolation of eel atrial natriuretic peptide. Zoological Science 14 993-999.

Tamura N, Ogawa Y, Yasoda A, Itoh H, Saito Y \& Nakao K 1996 Two cardiac peptide genes (atrial natriuretic peptide and brain natriuretic peptide) are organized in tandem in the mouse and human genomes. Fournal of Molecular and Cellular Cardiology 28 $1811-1815$

Tateyama H, Hino J, Minamino N, Kangawa K, Ogihara T \& Matsuo H 1990 Characterization of immunoreactive brain natriuretic peptide in human cardiac atrium. Biochemical and Biophysical Research Communications 166 1080-1087.

Togashi K, Kameya T, Ando K, Maruo F \& Kawakomi M 1991 Brain natriuretic peptides in human plasma, spinal cord and cerebrospinal fluid. Clinica Chimica Acta 201 193-200.

Ueda S, Sudoh T, Fukuda K, Kangawa K, Minamino N \& Matsuo H 1987 Identification of alpha atrial natriuretic peptide (4-28) and (5-28) in porcine brain. Biochemical and Biophysical Research Communications 149 1055-1062.

Wu F, Yan W, Pan J, Morser J \& Wu Q 2002 Processing of pro-atrial natriuretic peptide by corin in cardiac myocytes. Fournal of Biological Chemistry 277 16900-16905.

Yasuda-Kamatani Y \& Yasuda A 1999 Identification of orcokinin gene-related peptides in the brain of the crayfish Procambarus clarkii by the combination of MALDI-TOF and on-line capillary HPLC/Q-Tof mass spectrometries and molecular cloning. General and Comparative Endocrinology 118 161-172.

Received 15 September 2002 Accepted 14 March 2003 